\title{
Prognostic factors for gastroenteropancreatic neuroendocrine neoplasms (GEP-NENs): what's better?
}

\author{
Massimo Milione ${ }^{1}$
}

Received: 20 March 2017 / Accepted: 1 April 2017 / Published online: 17 April 2017

(C) Springer Science+Business Media New York 2017

Gastroenteropancreatic neuroendocrine neoplasms (GEPNENs) have been extensively studied in the effort to identify clinical, pathological and biological factors able to predict survival and risk of recurrence.

Undoubtedly, Ki67 has been considered among the most important prognostic factors in this setting since 1996, when La Rosa et al. [1] showed the predictive capability of $\mathrm{Ki} 67 \%$ counting alone in the nuclei of neoplastic cells. Therefore a $\mathrm{Ki67}>2 \%$ became the threshold to define prognosis in all subsequent WHO classifications to date [2]. Another "historical" prognostic factor is represented by morphology, defined as the grade of differentiation $(G)$ : this parameter allows distinguishing between well-differentiated GEP-NENs (WD-GEP-NENs) and poorly-differentiated ones (PD-GEP-NENs) [3].

The combined use of $\mathrm{Ki67}$ and morphology is not immediate in clinical practice. Actually, Ki67 has incorporated morphology, and indeed the 2010 WHO classification considers as well-differentiated only G1 $(\mathrm{Ki} 67<2 \%)$ and G2 NETs (Ki67 2-20\%), while GEP-NENs with Ki67> $20 \%$ are by definition poorly-differentiated [4, 5]. In recent years, this classification has been extensively revised with the proposal of new intermediate categories, such as the newly-proposed NET G3 [4, 5].

The integrated evaluation of $\mathrm{Ki} 67$ and morphology allows the full definition of prognosis for PD-GEP-NENs. On the other hand, it is not sufficient for WD-GEP-NENs,

Massimo Milione

Massimo.milione@istitutotumori.mi.it

1 Department of Pathology and Laboratory Medicine, 1st Division of Pathology, IRCCS Foundation National Cancer Institute, Milan, Italy and therefore the identification of other prognostic factors appears necessary. Indeed, WD-GEP-NENs are characterized by a wide spectrum of clinical presentations. On one side of the spectrum, we have "pseudo-benign" small gastric NENs with indolent behavior and no metastatic spread. On the opposite side, we find neoplasms with distant metastasis at diagnosis or liver involvement.

Prognostic factors evaluated to for WD-GEP-NENs include primary site (e.g., pancreatic disease is associated with a poorer prognosis) and tumor diameter, with size $>$ $2-3 \mathrm{~cm}$ being indicative of shorter survival [6, 7].

All the above-mentioned factors are however evaluable only after tumor resection, and therefore can only predict disease course. On the other hand, biological markers might provide early information on prognosis and even allow the estimation of the risk of neoplastic transformation [8]. Interestingly, while the prognostic factors for pancreatic WD-GEP-NENs were extensively investigated during the studies on the mTOR pathway [9], less extensive evidence is available for ileal disease although information on prognostic biomarkers is urgently required for these neoplasms. Indeed, differing from pancreatic disease, ileal WDGEP-NENs - presenting in almost all cases $\mathrm{Ki} 67<2 \%$ are classified as NET G1 according to the 2010 WHO classification, regardless of their clinical features. Therefore, the search for biological markers able to further stratify risk within this category may provide useful tools to guide clinical decisions. For ileal WD-GEP-NENs, the most intriguing biological markers are currently IMP3 and SDHB, which can predict malignant transformation.

IMP3 is a member of the insulinlike growth factor II mRNA binding protein (IMP) family that encloses also of IMP1 and IMP2 [10]. IMP3 is an oncoprotein constitutively expressed only during intrauterine life, when it promotes 
organ development. This protein is however expressed in some tumors, including lung and GEP NENs [8, 11, 12]. However, in the paper by Massironi et al. IMP3 expression has been found to be correlated with survival, although it is associated with more rapid metastatic spread [12].

Succinate Dehydrogenase subunit B (SDHB) is a mitochondrial protein with a major role in cellular respiration. Its abnormal expression (either decreased or increased) is a relevant prognostic factor for WD-NENs originating from small bowel [13] and predicts hepatic involvement in ileal WD-NENs [14].

Both IMP3 and SDHB have been extensively studied by immunohistochemistry (IHC), using ad hoc scores which include both quantitative (i.e., number of stained cells) and qualitative (i.e., staining intensity, compared with a control) evaluations [12-14]. However, in these studies the characteristics of the evaluated populations did not allow the use of quantitative assessments (e.g., polymerase chain reaction on next-generation sequencing platforms). This bias likely contributes to the poor predictive power of both IMP3 and SDHB when assessed alone. However, when IHC results are considered together with other prognostic factors they provide clinically relevant information. In lung PD-NENs, the expression of IMP3 is associated with more rapid recurrence than diseases not expressing this protein [11]. The combined evaluation of SDHB and Ki67 better predicts survival than either SDHB or Ki67 alone [13]. These studies are intriguing, since they pave the way for prospective investigations. In particular, the clinical relevance of both IMP3 and SDHB could be of most relevance if the evaluation of their expression in normal cells may help predict neoplastic transformation, with the possibility of direct assessment or in association with circulating microRNA [15]. While waiting for prospective investigations on IMP3 and SDHB to be completed, the evaluation of these markers, although indirect, may complement on the information provided by other well-established prognostic factors such as $\mathrm{Ki} 67$ and morphology.

Acknowledgements The author thanks Luca Giacomelli Ph.D for literature searches and English revision

\section{Compliance with ethical standards}

Conflict of interest The author declares that he has no competing interests.

\section{References}

1. S. La Rosa, F. Sessa, C. Capella, C. Riva, B.E. Leone, C. Klersy, G. Rindi, E. Solcia, Prognostic criteria in nonfunctioning pancreatic endocrine tumours. Virchows. Arch. 429(6), 323-333 (1996)
2. G. Rindi, R. Arnold, F.T. Bosman, C. Capella, D.S. Klimstra, G. Klöppel, P. Komminoth, E. Solcia. Nomenclature and classification of digestive neuroendocrine tumours. in World Health Organization Classification of Tumours, Pathology and Genetics of Tumours of the Digestive System. ed. by T.F. Bosman, F. Carneiro, R.H. Hruban, N.D. Theise (International Agency for Research on Cancer (IARC) Press, Lyon, 2010) pp. 10-12

3. C. Capella, P.U. Heitz, H. Höfler, E. Solcia, G. Klöppel, Revised classification of neuroendocrine tumours of the lung, pancreas and gut. Virchows. Arch. 425(6), 547-560 (1995)

4. N. Fazio, M. Milione, Heterogeneity of grade 3 gastroenteropancreatic neuroendocrine carcinomas: new insights and treatment implications. Cancer. Treat. Rev. 50, 61-67 (2016)

5. M. Milione, P. Maisonneuve, F. Spada, A. Pellegrinelli, P. Spaggiari, L. Albarello, E. Pisa, M. Barberis, A. Vanoli, R. Buzzoni, S. Pusceddu, L. Concas, F. Sessa, E. Solcia, C. Capella, N. Fazio, S. La Rosa, The clinicopathologic heterogeneity of Grade 3 Gastroenteropancreatic neuroendocrine Neoplasms: morphological differentiation and proliferation identify different prognostic categories. Neuroendocrinology 104(1), 85-93 (2017)

6. F. Panzuto, S. Nasoni, M. Falconi, V.D. Corleto, G. Capurso, S. Cassetta, M. Di Fonzo, V. Tornatore, M. Milione, S. Angeletti, M. S. Cattaruzza, V. Ziparo, C. Bordi, P. Pederzoli, G. Delle Fave, Prognostic factors and survival in endocrine tumor patients: comparison between gastrointestinal and pancreatic localization. Endocr. Relat. Cancer 12(4), 1083-1092 (2005)

7. U.F. Pape, U. Berndt, J. Müller-Nordhorn, M. Böhmig, S. Roll, M. Koch, S.N. Willich, B. Wiedenmann, Prognostic factors of long-term outcome in gastroenteropancreatic neuroendocrine tumours. Endocr. Relat. Cancer 15(4), 1083-1097 (2008)

8. P. Vercellini, F.M. Cribiù, A. Del Gobbo, M.L. Carcangiu, E. Somigliana, S. Bòsari, The oncofetal protein IMP3: a novel biomarker and triage tool for premalignant atypical endometriotic lesions. Fertil. Steril. 99(7), 1974-1979 (2013)

9. M. Falconi, D.K. Bartsch, B. Eriksson, G. Klöppel, J.M. Lopes, J. M. O'Connor, R. Salazar, B.G. Taal, M.P. Vullierme, D. O'Toole; Barcelona Consensus Conference participants, ENETS consensus guidelines for the management of patients with digestive neuroendocrine neoplasms of the digestive system: well-differentiated pancreatic non-functioning tumors. Neuroendocrinology. 95(2), 120-134 (2012)

10. J. Nielsen, J. Christiansen, J. Lykke-Andersen, A.H. Johnsen, U.M. Wewer, F.C. Nielsen, A family of insulin-like growth factor II mRNA-binding proteins represses translation in late development. Mol. Cell. Biol. 19(2), 1262-1270 (1999)

11. A. Del Gobbo, V. Vaira, E. Guerini Rocco, A. Palleschi, G. Bulfamante, D. Ricca, S. Fiori, S. Bosari, S. Ferrero, The oncofetal protein IMP3: a useful marker to predict poor clinical outcome in neuroendocrine tumors of the lung. J. Thorac. Oncol. 9 (11), 1656-1561 (2014)

12. S. Massironi, A. Del Gobbo, F. Cavalcoli, S. Fiori, D. Conte, A. Pellegrinelli, M. Milione, S. Ferrero, IMP3 expression in smallintestine neuroendocrine neoplasms: a new predictor of recurrence. Endocrine (2017). doi:10.1007/s12020-017-1249-x

13. M. Milione, S. Pusceddu, P. Gasparini, F. Melotti, P. Maisonneuve, V. Mazzaferro, F.G. de Braud, G. Pelosi, Succinate dehydrogenase B subunit immunohistochemical expression predicts aggressiveness in well differentiated neuroendocrine tumors of the ileum. Cancers 4(3), 808-820 (2012)

14. M. Milione, P. Maisonneuve, A. Pellegrinelli, S. Pusceddu, G. Centonze, F. Dominoni, C. Brambilla, M. Rubino, A. Faggiano, R. Buzzoni, L. Concas, L. Giacomelli, J. Coppa, V. Mazzaferro, F. de Braud Loss of succinate dehydrogenase subunit B (SDHB) as a prognostic factor in advanced ileal well-differentiated 
neuroendocrine tumors. Endocrine (2016). doi:10.1007/s12020016-1180-6

15. E. Patterson, R. Webb, A. Weisbrod, B. Bian, M. He, L. Zhang, A.K. Holloway, R. Krishna, N. Nilubol, K. Pacak, E. Kebebew,
The microRNA expression changes associated with malignancy and SDHB mutation in pheochromocytoma. Endocr. Relat. Cancer 19(2), 157-166 (2012) 